Ann. Génét. Sél. anim., I970, 2 (I), 75-84.

\title{
EFFETS D'HÉTÉROSIS SUR LES POIDS A LA NAISSANCE ET AU SEVRAGE DANS LE CROISEMENT CHAROLAIS $\times$ ABERDEEN ANGUS
}

\author{
Héctor Ariel MOLINUEVO (*) \\ Instituto nacional de Tecnologia agropecuaria \\ Balcarce (Argentine)
}

RÉSUMÉ

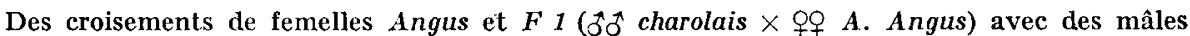
Angus, charolais et $F 1$ ont permis d'estimer la vigueur hybride en comparant pour chaque groupe de mères (Angus et $F$ 1) les résultats des taureaux $F 1$ avec la moyenne pondérée des résultats des autres taureaux (Angus et charolais). La vigueur hybride ainsi estimée est importante.

\section{INTRODUCTION}

Les croisements entre les races à viande anglo-saxonne et le Charolais ont pris une grande extension au cours des dernières années. Néanmoins très peu d'études réalisées sur ces croisements permettent une estimation de l'hétérosis indépendamment de 1'effet maternel en raison de l'effectif très limité des femelles charolaises disponibles dans les pays intéressés. En outre, comme l'effet maternel peut être considérable sur le poids à la naissance et au sevrage, il ne semble pas possible, pour l'instant, d'estimer valablement la vigueur hybride en l'absence de croisements réciproques.

En complément des travaux effectués en Argentine (Lopez SaUbidet et al., I963) sur l'intérêt de la race charolaise en croisement de I $^{\text {re }}$ génération, on s'est précisément intéressé à l'estimation des effets non additifs intervenant dans ces croisements : estimation particulièrement importante en vue d'orienter les

$\left(^{*}\right)$ Adresse actuelle : Station centrale de génétique animale, Centre national de Recherches zootechnique, 78 - Jouy-en-Josas. Institut national de la Recherche agronomique. 
programmes d'amélioration génétique. On a utilisé dans ce but des vaches $A b e r-$ deen-Angus (A) et $F_{I}$ (ơ charolais $\times$ of Aberdeen Angus) d'une part, des taureaux $A, F_{I}$ et charolais (C) d'autre part. Dans chaque groupe de mères on compare les performances des animaux issus des taureaux $F_{I}$ avec celles des produits des taureaux $A$ et $C$.

De tels accouplements permettent de réaliser une estimation minimum de la vigueur hybride correspondant au croisement $C \times A$. La présente étude rassemble les résultats partiels relatifs au poids à la naissance et au poids au sevrage.

\section{MATÉRIEL ET MÉTHODES}

Les effectifs de veaux nés 'par type d'accouplement réalisé figurent sur le tableau I. On a utilisé six taureaux de chaque race chaque année; les taureaux $A$ et $F 1$ étaient produits en Argentine tandis que les taureaux $C$ étaient représentés par de la semence importée de France et issue d'un nombre équivalent de mâle en cours de contrôle de descendance. On a observé au total 350 veaux à la naissance correspondant à trois saisons de vêlage et 97 veaux au sevrage relatifs à deux saisons seulement. Dans le cas des accouplements $\delta \delta^{\top} F 1 \times$ 우 $A$ on ne disposait en fin que de deux saisons de vêlage pour le poids à la naissance; les données relatives au poids au sevrage étaient en nombre trop limité pour permettre une conclusion valable.

\section{TABLEAU I}

Répartition des vêlages

\begin{tabular}{|c|c|c|c|c|c|c|c|c|c|c|c|c|c|}
\hline \multicolumn{5}{|c|}{ Année } & 1966 & 1967 & 1968 & 1969 & 1967 & I 968 & 1966 & I 967 & I968 \\
\hline 오 & & & & & \multicolumn{3}{|c|}{$A$} & \multicolumn{3}{|c|}{$F_{I}$} & \multicolumn{3}{|c|}{$C$} \\
\hline$A$ & $\begin{array}{l}\text { Nombre de vêlages } \\
\text { Vêlages aidés . . . } \\
\text { Veaux morts. . . . }\end{array}$ & ${ }^{\circ}$ & . & . & $\begin{array}{r}\text { I } 8 \\
\text { O } \\
\text { I }\end{array}$ & $\begin{array}{r}30 \\
0 \\
0\end{array}$ & $\begin{array}{r}25 \\
0 \\
\mathbf{I}\end{array}$ & & $\begin{array}{r}34 \\
1 \\
2\end{array}$ & $\begin{array}{r}23 \\
\mathbf{I} \\
\mathrm{I}\end{array}$ & $\begin{array}{r}23 \\
1 \\
2\end{array}$ & $\begin{array}{r}30 \\
1 \\
4\end{array}$ & $\begin{array}{r}26 \\
1 \\
2\end{array}$ \\
\hline$F_{I}$ & $\begin{array}{l}\text { Nombre de vêlages } \\
\text { Vêlages aidés . . . } \\
\text { Veaux morts. . . . }\end{array}$ & • & . & . & $\begin{array}{r}23 \\
1 \\
3\end{array}$ & $\begin{array}{r}18 \\
0 \\
0\end{array}$ & $\begin{array}{r}20 \\
0 \\
\mathrm{I}\end{array}$ & $\begin{array}{r}2 \mathrm{I} \\
\mathrm{I} \\
2\end{array}$ & $\begin{array}{r}2 \mathrm{I} \\
\mathrm{I} \\
2\end{array}$ & $\begin{array}{r}19 \\
1 \\
2\end{array}$ & $\begin{array}{l}3 \mathrm{I} \\
\mathrm{I} 2 \\
2 \mathrm{I}\end{array}$ & $\begin{array}{r}25 \\
5 \\
4\end{array}$ & $\begin{array}{r}3 \mathbf{I} \\
\mathbf{0} \\
0\end{array}$ \\
\hline
\end{tabular}

Les vêlages ont eu lieu chaque année pendant une période de deux mois et demi; on a enregistré les cas d'assistance au vêlage et de mortalité ainsi que le poids à la naissance des veaux (pris dans un délai maximum de 14 heures si la naissance est pendant le soir ou la nuit, et de 3 heures si elle est pendant la journée). Les mâles sont castrés dès la 1 re semaine, le sevrage intervient à $240 \mathrm{j}$. $\pm 7 \mathrm{j}$., le poids au sevrage est ajusté à l'âge moyen correspondant par intrapolation linéaire entre les poids à la naissance et au sevrage.

Au début de l'expérience les vaches $A$ avaient déjà effectué au moins un vêlage; les femelles F1 étaient des primipares. Pour cette raison les résultats des deux groupes de vaches ne sont pas comparables entre eux; on fera donc une analyse séparée pour chaque groupe. On a aussi analysé en raison de leur incidence pratique ıes dífficultés liées au vêlage, de ces femelies dans 
le cas des trois types d'accouplements. En raison de la faible fréquence des difficultés observées dans l'un des groupes comparés et du faible nombre d'observations, on a exprimé l'intervalle de confiance de cette fréquence au seuil de probabilité $1-\alpha$ :

$$
1-\alpha=\mathrm{P}\left[\hat{p}-a \sqrt{\frac{p q}{n}}<p<\hat{p}+a \sqrt{\frac{p q}{n}}\right]
$$

selon une relation définie par LEFort (1967):

$$
\frac{(\hat{p}-p)^{2}}{p q / n}<a^{2}
$$

avec $\hat{p}=$ fréquence estimée

$p=$ fréquence théorique

$q=1-p$

$n=$ nombre d'observations

$a=$ valeur ayant la probabilité d'être dépassée en valeur absolue par une variable normale réduite.

En vue de tester la signification statistique de l'efiet d'hétérosis sur les poids à la naissance et au sevrage, on compare à 0 , pour chaque groupe de femelles, la différence (D) entre la moyenne des veaux issus des taureaux $F 1(\mathrm{Cr}=$ "groupe croisé $")$ et celle des deux autres groupes issus des taureaux charolais et Aberdeen Angus ("groupes parentaux $\mathrm{P}_{1}$ et $\left.\mathrm{P}_{2}\right) \mathrm{D}=\mathrm{Cr}-\frac{1}{2}\left(\mathrm{P}_{1}+\mathrm{P}_{2}\right)$.

On considère qu'il y a hétérosis si $\mathrm{D} \neq 0$; par contre si $\mathrm{D}=0$ on ne peut conclure car les groupes parentaux, $P_{1}$ et $P_{2}$, pris comme base de comparaison peuvent exprimer eux-mêmes un effet d'hétérosis. Ainsi, on cherche ce que nous appelons hétérosis "minimum ", car il y a. une certaine portion d'hétérosis qu'on ne peut pas détecter.

Le test statistique est obtenu en calculant $t=\frac{\mathrm{D}}{\sigma \mathrm{D}}$ qui peut être considéré comme distribué normalement lorsque, comme c'est le cas pour le poids à la naissance, le nombre d'observations est supérieur à 60 . En pratique, la valeur $D$ est calculée en cumulant les différences ci-dessus relatives à chaque sexe dans chaque année et en les pondérant par l'inverse de leurs variances (on effectue une combinaison linéaire des différences moyennes élémentaires de variances minimum) :

$$
\mathrm{D}=\sum_{i} \frac{\frac{d_{i}}{\sigma_{i}^{2}}}{\Sigma \frac{1}{\sigma_{i}^{2}}}
$$

avec $\mathrm{d}_{i}=$ différence moyenne relative à la ième combinaison sexe $\times$ années

$\sigma_{i}^{2}=$ variance de cette différence.

Un test global de vigueur hybride a été établi à partir des mères $A$ et $F 1$ en combinant toutes les probabilités $\left(\mathrm{P}_{m}\right)$ liées aux différences $D_{m}$ enregistrées. On s'est servi du test décrit par Kendall (1946) : $\mathrm{M}^{2}=2 \sum_{m} \log _{e} p_{m}$, ou $p_{m}$ est la probabilité liée à chaque situation $M^{2}$ étant distribué comme $\chi^{3}$.

\section{RÉSULTATS}

\section{a) Parturition}

Les fréquences des vêlages difficiles, de mortalité (comprenant en fait la mortinatalité et la mortalité périnatale étendue à la première semaine) ainsi que les intervalles de confiance correspondants sont rassemblés sur le tableau 2 pour les accouplements concernant les génisses $F_{I}$ qui étaient les seuls à présenter des difficultés de vêlage avec une fréquence notable.

On observe que les difficultés de vêlage et les cas de mortalité des veaux liés aux traumatismes consécutifs à ces vêlages sont importants quand ces génis- 
ses sont accouplées à des taureaux charolais. Les fréquences de ces incidents sont par contre faibles et comparables sur les deux types d'accouplements : génisses $F_{I} \times$ taureaux $A$ ou $F_{I}$.

TABLEAU 2

Fréquence des vêlages difficiles et des cas de mortilatalité des veaux chez les génisses $F_{I}$

\begin{tabular}{|c|c|c|c|c|}
\hline \multirow{2}{*}{$\begin{array}{c}\text { Type } \\
\text { d'accouplement }\end{array}$} & \multicolumn{2}{|c|}{ vêlages difficiles } & \multicolumn{2}{|c|}{ Veaux morts } \\
\hline & fréquence & $\begin{array}{l}\text { intervalle de } \\
\text { confiance }\end{array}$ & fréquence & $\begin{array}{l}\text { intervalle de } \\
\text { confiance }\end{array}$ \\
\hline $\begin{array}{l}A \times F I \\
F I \times F I\end{array}$ & 0,04 & 0,$02 ; 0,1$ I & $0, \mathbf{I} \mathbf{I}$ & 0,$06 ; 0,19$ \\
\hline$C \times F I$ & 0,39 & 0,$29 ; 0,45$ & 0,68 & 0,$56 ; 0,76$ \\
\hline
\end{tabular}

b) Hétérosis sur les poids à la naissance et au sevrage

\section{Poids à la naissance}

Les différences, $\mathrm{D}=\mathrm{Cr}-1 / 2\left(\mathrm{P}_{1}+\mathrm{P}_{2}\right)$, entre le " groupe croisé " et les " groupes parentaux " figurent sur le tableau 3 pour les femelles $A$ et $F_{I}$.

Lorsque l'on considère les mères $A$, la valeur de $\mathrm{D}$ n'est pas significativement différente de 0 ; on enregistre, en fait, un faible écart négatif pour les mâles et positif pour les femelles. Par contre, dans le cas des mères $F_{I}$, l'hypothèse d'un effet d'hétérosis doit être acceptée avec une très faible probabilité d'erreur.

Il est difficile de donner une explication biologique à cette différence de comportement entre les mères $A$ et $F_{I}$; peut-être s'agit-il tout simplement d'un phénomène lié à l'échantillonnage.

La signification de la différence entre les deux échantillons de mères a été testée suivant le critère $M^{2}$ décrit ci-dessus. La valeur obtenue $\mathbf{M}^{2}=39,82$ correspond à $\chi^{2}(8 ; 0,01)=20.09$ ce qui conduit à considérer comme hautement probable l'hypothèse d'un effet d'hétérosis.

On constate, enfin, que l'hétérosis est plus grand chez les femelles que chez les mâles à la fois pour les mères $A$ et $F_{I}$, ce qui est en accord avec l'hypothèse de STONAKER (I963). 
TABIEAU 3

Nombre d'observations $(\mathbf{n})$, moyennes $(\overline{\mathbf{x}})$ des poids à la naissance et différences liées à l'hétérosis $(D)$

\begin{tabular}{|c|c|c|c|c|c|c|c|c|}
\hline \multicolumn{3}{|c|}{ Sexe des produits } & \multicolumn{3}{|c|}{ Mâles } & \multicolumn{3}{|c|}{ Femelles } \\
\hline père & \multicolumn{2}{|c|}{ Année de vêlage } & I966 & I967 & 1968 & I966 & I 967 & I 968 \\
\hline \multirow{2}{*}{$A$} & \multirow{2}{*}{$A$} & $n$ & & 14 & 9 & & 16 & I6 \\
\hline & & $\overline{\mathbf{x}}$ & & 29,6 & 27,4 & & 26,8 & 25,2 \\
\hline \multirow{2}{*}{$F_{I}$} & \multirow{2}{*}{$A$} & $n$ & & 20 & I I & & I 4 & I 2 \\
\hline & & $\overline{\mathbf{x}}$ & & $33, I$ & 32,5 & & $3 \mathrm{I}, 2$ & $3 \mathrm{I}, 4$ \\
\hline \multirow{2}{*}{$C$} & \multirow{2}{*}{$A$} & $n$ & & I 7 & I 8 & & I3 & 8 \\
\hline & & $\overline{\mathbf{x}}$ & & $3^{8,9}$ & $3^{8,4}$ & & 36,0 & 33,2 \\
\hline \multicolumn{3}{|c|}{$\mathrm{D}$} & \multicolumn{3}{|c|}{$-\mathbf{I}, 6$} & \multicolumn{3}{|c|}{$+1,7$} \\
\hline \multicolumn{3}{|c|}{$\mathrm{D} \%$} & \multicolumn{3}{|c|}{$-4,8$} & \multicolumn{3}{|c|}{5,8} \\
\hline \multirow{2}{*}{$A$} & \multirow{2}{*}{$F_{I}$} & $n$ & I I & I I & I 3 & I 2 & 7 & 7 \\
\hline & & $\overline{\mathbf{x}}$ & 32,9 & 32,4 & $3^{2,5}$ & 27,5 & 32,4 & $3 \mathrm{I}, 7$ \\
\hline \multirow{2}{*}{$F_{I}$} & \multirow{2}{*}{$F_{I}$} & $n$ & I 2 & I3 & 8 & 9 & 8 & I I \\
\hline & & $\overline{\mathbf{x}}$ & 37,8 & $3^{8}, 7$ & 38,9 & 35,9 & 36,2 & 39,0 \\
\hline \multirow{2}{*}{$C$} & \multirow{2}{*}{$F_{I}$} & $n$ & 9 & II & Io & 5 & I 4 & II \\
\hline & & $\bar{x}$ & $39, \mathrm{r}$ & 43,7 & $3^{8,6}$ & $3^{8,7}$ & $4 I, 7$ & 39,2 \\
\hline \multicolumn{3}{|c|}{$\mathrm{D}$} & \multicolumn{3}{|c|}{$3,8^{* *}$} & \multicolumn{3}{|c|}{$4,5^{* *}$} \\
\hline \multicolumn{3}{|c|}{ D \% } & \multicolumn{3}{|c|}{ I0,5 } & \multicolumn{3}{|c|}{12,9} \\
\hline
\end{tabular}

Différences significatives au seuil de probabilité de $0,0 x$.

Poids au sevrage

Comme nous l'avons déjà signalé, seules ont été analysées suivant le même processus les données relatives aux veaux issus des mères $F_{I}$ et ceci sur les deux années. Toutefois en raison du nombre plus restreint de données, les conditions d'application du test $\mathrm{M}^{2}$ (distribution normale de $t=\frac{\mathrm{D}}{\sigma \mathrm{D}}$ ) ne sont pas exactement remplies : l'approximation qui en résulte augmente le risque de $\mathrm{I}^{\mathrm{re}}$ espèce.

Les résultats obtenus ont été néanmoins présentés sur le tableau 4. La valeur trouvée pour $\mathrm{M}^{2}=27,78$ correspond à $\chi^{2}{ }_{(4 ; 0,01)}=13.28$. On peut là aussi conclure, malgré les limitations théoriques précédentes, à un effet d'hétérosis.

Contrairement à ce qui a été observé pour le poids à la naissance, les mâles semblent présenter un hétérosis supérieur à celui des femelles. 
TABLEAU 4

Nombre d'observations $(\mathrm{n})$, moyennes $(\overline{\mathbf{x}})$ et différences liées à l'hétérosis $(D)$ pour le poids au sevrage

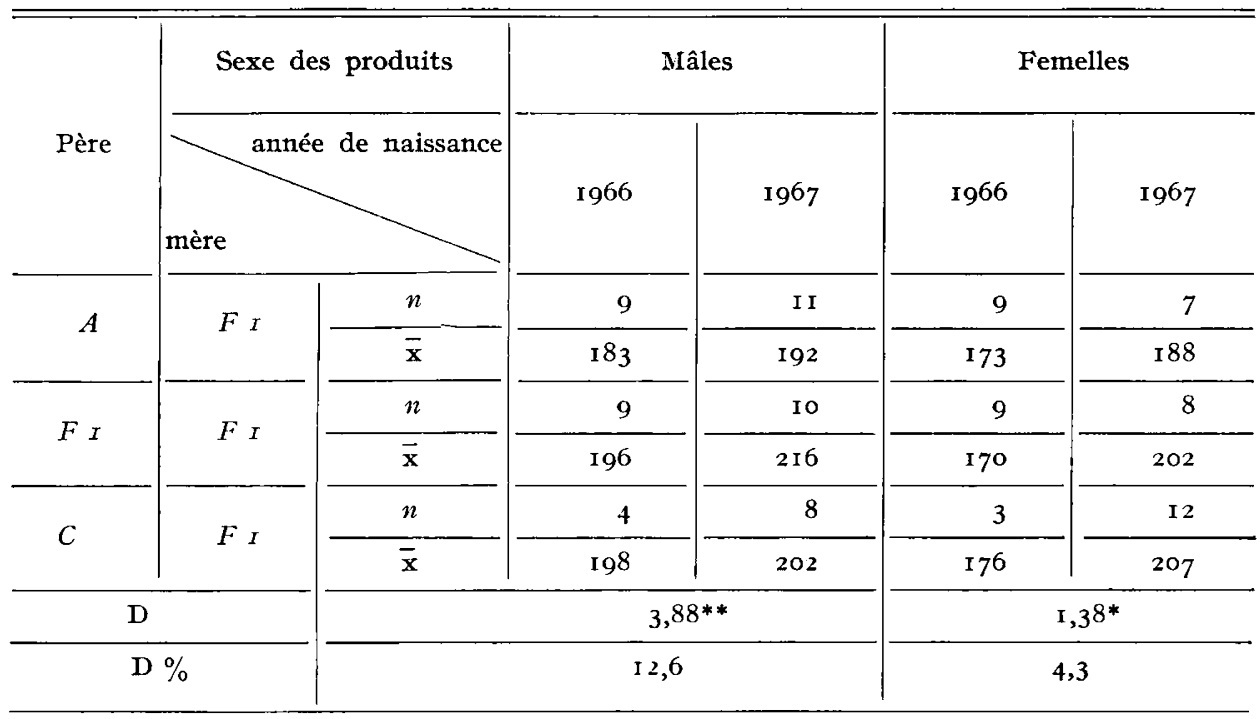

* Différence significative au seuil de probabilité de 0,05 .

** Différence significative au seuil de probabilité de o,or.

\section{DISCUSSION ET CONCLUSION}

\section{a) Parturition}

Les difficultés observées dans les accouplements $C \times$ génisses $F_{I}$ peuvent être liées à un développement insuffisant du bassin de ces femelles par rapport au poids élevé des veaux. Nos résultats sont en accord à cet égard avec ceux trouvés par Lopez SAUbidet et al. (I963) et par SAGebiel et al. (I969) qui ont travaillé sur femelles Angus croisées avec des mâles charolais. SAGEBIEI et al. trouvent une fréquence de vêlages difficiles de $5^{8} \mathrm{p}$. Ioo pour les femelles primipares de race $A n g u s$; ce chiffre est légèrement supérieur à celui que nous avons trouvé pour les femelles $F_{I}$ au premier vêlage. MonTEIRo (r969) signale de son côté la relation étroite qui existe entre les poids du veau et les difficultés de vêlages à partir de femelles Frisonnes, Ayrshire et Jersey. Il trouve également une diminution importante de la fréquence des vêlages difficiles en deuxième mise-bas, par rapport à la première.

Les difficultés de vêlages et la mortalité des veaux issus du croisement : ơocharolais $\times$ génisses $F_{I}$ interdisent de recommander l'utilisation d'un tel type de croisement dans la pratique. Au contraire, ce croisement semble intéressant avec des femelles $F_{I}$ au deuxième vêlage. 


\section{b) Hétérosis}

Si on compare nos résultats avec ceux qui, dans la bibliographie (tableau 5) se rapportent à des croisements réciproques entre le charolais et les races anglosaxonnes (SAGeBiel et al., I967 $a$ et $b$; KLOSTERman et al., I968 et PAHNish et al., I969), on constate que certaines de nos estimations figurent parmi les plus élevées; c'est le cas en particulier pour les estimations de vigueur hybride relatives au poids :

a) à la naissance des produits des mères $F r$.

b) au sevrage des veaux mâles issus du même groupe de mères.

\section{TABLEAU 5}

Valeurs extrêmes de vigueur hybride pour le poids à la naissance et au sevrage (Expériences comportant des croisements réciproques exclusivement)

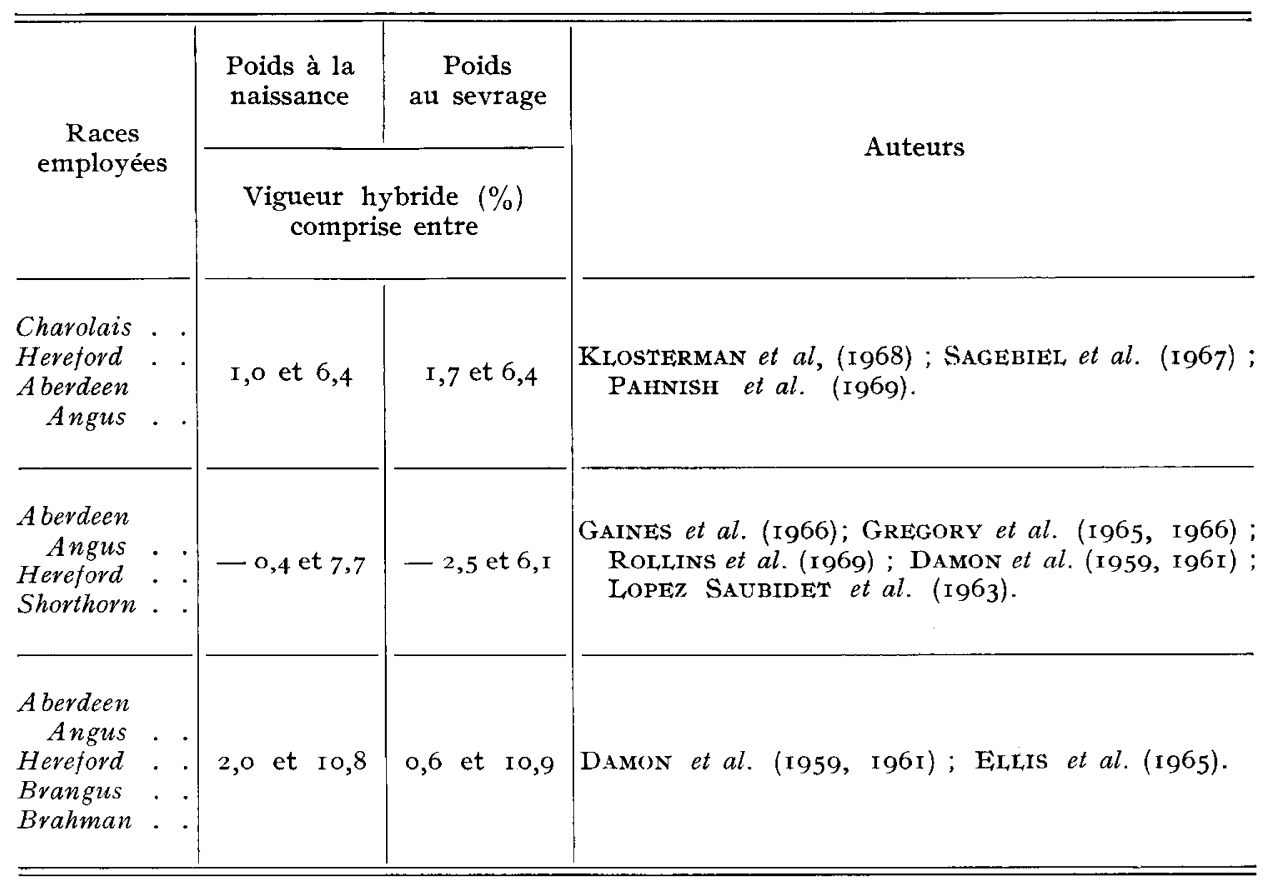

Ce n'est qu'avec les croisements entre Brahman et Hereford ou entre Brahman et $A n g u s$ que 1'on trouve des effets d'hétérosis du même ordre de grandeur que ceux que nous enregistrons (DAmon et al., I959 et I96r; Eirlis et al., I965).

En fait, la valeur élevée de nos estimations prend toute sa signification si on considère que les comparaisons effectuées sous-estiment la vigueur hybride réelle, les " groupes parentaux " étant constitués d'animaux qui peuvent exprimer eux-mêmes des effets d'hétérosis. 
Néanmoins, on peut penser à priori qu'il y aura davantage de vigueur hybride dans le croisement Charolais $\times$ races anglo-saxonnes que dans le cas d'un croisement entre ces dernières races; cela s'expliquerait par les différences d'origine, de format et de structure corporelle qui existent entre ces deux types d'animaux. Dans ces conditions, il nous parait vraisemblable de trouver des estimations de la vigueur hybride plus élevées que celles qui ont été établies dans le cas du croisement entre races anglo-saxonnes par différents auteurs (DAMON et al., I959 et I96I; LOPEZ SAUBIDET et al., I963; GREGORy et al., I965 et I966; GAINES et al., I966; Rolisivs et al., I969). Par contre, les résultats de SAGEBIEL et al., (I967); Klosterman et al., (I968) et Pahnish et al., (I969), relatifs au croisement Charolais $\times$ races anglo-saxonnes, sont du même ordre de grandeur que ceux des auteurs que nous venons de citer.

Certaines justifications peuvent être invoquées pour expliquer les divergences observées :

a) Nous avons travaillé avec de la semence de taureaux charolais directement importée de France tandis que les expériences faites au États-Unis concernaient des taureaux Charolais issus de croisements d'absorption avec des mères d'origines variées. Les différences entre les deux types de Charolais peuvent être assez importantes pour intervenir dans l'estimation des effets d'hétérosis.

b) La vigueur hybride dans le cas du croisement Charolais $\times$ Aberdeen Angus peut être plus élevée que dans le cas du croisement Charolais $\times$ Hereford (quoi que les résultats de PaHnish et al., I969, soient d'une tendance contraire). Si cette hypothèse est vraie, elle explique le fait que nos estimations sont plus élevées que celles de SAGEBIEL et al., (Ig67 $a$ et $b$ ) qui donnent les résultats moyens des croisements réciproques suivants : Charolais $\times$ Aberdeen Angus, Charolais $\times$ Hereford et Aberdeen Angus $\times$ Hereford et celles de KLosterman et al., (I968) qui ont étudié le croisement Charolais $\times$ Hereford.

c) Il peut également exister des différences génétiques entre les troupeaux d'origine des mères en Argentine et aux Etats-Unis.

d) La mortalité des veaux nés de pères Charolais entraîne une sélection des veaux survivants, les veaux les plus lourds ayant plus de chances de mourir au vêlage. Dans notre expérience ce fait devrait conduire à surestimer la vigueur hybride. Par contre dans les croisements réciproques classiques tels que ceux mentionnés ci-dessus, si ce phénomène de mortalité des veaux les plus lourds apparaissait, 1a vigueur hybride aurait été de ce fait sous-estimée. Néanmoins les valeurs d'hétérosis que nous avons obtenues ne semblent pas plus élevées l'année où la mortalité des veaux a été la plus importante.

e) Enfin, les milieux sur lesquels toutes ces expériences ont été faites sont très différents; or, les variations de milieu peuvent expliquer les divergences constatées entre les résultats, notamment s'il existe pour les caractères étudiés des interactions génotype $\times$ milieu importantes.

Tout en tenant compte des résultats antérieurs nous devons donc conclure à un effet d'hétérosis important dans les conditions et les types de croisements avec lesquels nous avons travaillé. 
Il convient enfin de signaler que le procédé utilisé pour mettre en évidence la présence d'hétérosis semble être intéressant dans plusieurs cas où les croisements réciproques sont difficiles à réaliser, du fait du faible nombre et $d u$ coût élevé des femelles de race étrangère.

Rę̧u pour publication en janvier I97o.

\title{
REMERCIEMENTS
}

Je tiens à remercier MM. Rouvier, B. Vissac, R. et C. Legault, Station centrale de Génétique animale, CNRZ pour leurs suggestions et leurs critiques sur l'analyse et la rédaction de ce travail dont l'éxécution nous a été facilitée par la collaboration de MM. R. Sullivan et R. Sofiak (I.N.T.A., Balcarce).

\section{SUMMARY}

\author{
HETEROSIS EFFECTS ON BIRTH AND WEANING WEIGHTS IN CROSSES \\ OF CHAROLAIS $\times$ ABERDEEN ANGUS
}

It has been made an application of contemporary comparison to estimate the hybrid vigour in the mating of females Aberdeen Angus and $F 1$ (Charolais $\times$ Aberdeen Angus) with males Aberdeen Angus, $F 1$ and Charolais.

Because the difference in age between the two groups of dams (A. Angus and $F 1$ ) each group is analyzed separately. Only the values of probability linked with each estimation are pooled for an overall test of heterosis.

In its simplest term, there are heterosis if $\mathrm{D}=\mathrm{Cr}-{ }^{1} /{ }_{2}\left(\mathrm{P}_{1}+\mathrm{P}_{2}\right) \neq 0$, where $\mathrm{Cr}=$ mean of the progeny of $F 1$ sires; $P_{1}$ and $P_{2}=$ means of the progeny of Angus and Charolais sires, respectively. On the contrary, if $\mathrm{D}=0$ it is not possible to conclude that heterosis is absent. The heterosis estimated is, in fact, due to recombinations and associations of chromosomes in the progeny of $\mathrm{F} 1$ sires, rising epistatic effects which are not possibles in the $\mathrm{F} 1$ generation, nor they are in the progeny of the Angus or Charolais sires.

The estimated hybrid vigour is of the order of $-4,8 \%$ and $5,8 \%$ for the birth weight of males and females respectively, out of $A$. Angus dams, and 10,5\% and $12,9 \%$ for the same weight of males and females out of $F 1$ dams. For weaning weight, the hybrid vigour of males and females, respectively, was of the order of $12,6 \%$ and $4,3 \%$ for the progeny of $F 1$ females.

When the probability values were conveniently pooled for an overall test of heterosis, the hypothesis of its presence seems to be acceptable with very little risk of error.

\section{RÉFÉRENCES BIBLIOGRAPHIQUES}

Damon R. A. jr, Mc Craine S.E., Crown R.M., Singletary C.B., I959. Performance of crossbred beef cattle in the Gulf Coast Region. J. animal. Sci., 18, 437-447.

Damon R.A. jr., Harvey W.R., Sngletary C.B., McCraine S.E., Crown R.M., I96x. Genetic analysis of crossbreding beef cattle. J. animal. Sci., 4, 849-857.

Ellis G.F. jr., Cartwrght T.C., KRUSE W.E., r965. Heterosis for birth weight in Brahman - Hereford crosses. J. animal. Sci., 24, 93-96.

Gaines J.A., McClure W.H., Vogt D.W., Carter R.C., KINkaID C.M., I966. Heterosis from crosses among british breeds of beef cattle : fertility and calf performance to weaning. J. animal. Sci., 25, 5-13.

Gregory K.E., Swiger L.A., Koch R.M., Sumption I.J., Rowden W.W., Ingal.ls J.E., I965. Heterosis in preweaning traits of beef cattle. J. animal Sci., 24, 2I-28.

Gregory K.E., Swiger L.A., Koch R.M., Sumption L.J., INGalls J.E., Rowden W.W. RothlisberGER J.A. I966 a. Heterosis effects on growth rate of beef heifers. J. animal. Sci., 25, $290-298$. 
Gregory K.E., Swiger I.A., Sumption I.J., Koch R.M., INGalls J.E., Rowden W.W., RothlisberGER J.A., I 966 b. Heterosis effects on growth rate and feed efficiency of beef steers. $J$. animal. Sci., 25 299-310.

KENDALI M.G., 1946. The advance theory of statistics, vol. 2, 132-133, Charles Grifrin and company limited, London.

Klosterman E.W., Camill V.R., Parkek C.F., r968. A comparison of the Hereford and Charolais breeds and heir crosses under two systems of management. Res. Bull., 1011, Ohio Agr. Res. and Dev. Center Wooster, Ohio.

LEFORT G., 1967. Mathématiques pour les sciences biologiques et agronomiques, 554-555, Armand CoLIN, Paris.

lopez Saubidet C., Cavandoli C.H., Igartua O.A., Joandet G.E., Cabrini E.J., Villar J.A., Sivori I.H., Hernandez O., Covas G., Kugler W.F., I963. Cruzas con Charoles en la Region Pampeana. Bol. Técnico (6), Estación Experimental Balcarce, Balcarce Argentine.

Monteiro L.S., I969. The relative size of calf and dam and the frequency of calving difficulies. Animal Prod., 11, 293-306.

Pahnish O.F., Brinks J.S., URICK J.J., KNAPP B.W., RIIEY T.M., I969. Results from crossing beef $x$ beef and beef $x$ dairy breeds calf performance to weaning. $J$. animal. Sci., 28, $291-299$.

Rollins W.C., Loy R.G., CARol. F.D., WAgnon K.A., 1969. Heterotic effects in reproduction and growth to weaning in crosses of the Angus, Hereford and Shorthorn breeds. J. animal. Sci., 28, 43 I-436.

Sagebiel J.A., Langford L.L., Sibbit W.R., Comfort J.E., Dyer A.J., L.ASley J.F., r967 a. Heterosis in preweaning traits in beef cattle. J. animal. Sci., 26, 888.

Sagebiel J.A., Sibbit W.R., I.ANgFord L.I., Dyer A.J., Comfort J.E., L.ASley J.F., i967 b. Heterosis in weaning, postweaning and slaughter traits of beef heifers. J. animal. Sci., 25, 889 .

STONAKer H.H., I963. A genetic hypothesis for sex mating systems interactions in growth of cattle and poultry. J. animal., Sci., 22, 320-325. 